and courage of these men and women, the finding for them, or the creation for them, of a useful existence, when unaided they are doomed to lives of idleness, uselessness and despair. It certainly presents great possibilities of increased efficiency in our present system.

We have had examples of the possibilities in the institutions for the blind, Dr. Hall's pottery at Marblehead, and Dr. Jaeger's Hospital of Hope in New York. All of those who have had much to do with hospitals will recognize the need.

I wish further to make the point that this work should not be based on appeals to the sympathy of employers, but on demonstrating or developing the fitness of the individual for the job. Sympathy employers may have, but they cannot be expected to clog their machinery with the unfit. I cannot at this time go into the subject at greater length. I wish, however, to urge the importance of this subject.

These are a few of the problems still awaiting solution and they loom up large. Ten years ago a hospital authority remarked that it seemed to him that most of the hospital problems had been settled. I hardly think we could agree with him, even now, only ten years later, for as I see it, the modern hospital is becoming an increasingly important factor in every community, both in the preservation of health and as an educational institution.

\section{THE ESSENTIAL FACTORS IN THE DIAG- NOSIS OF CHRONIC GASTRIC AND DUODENAL ULCERS *}

\author{
GEORGE B. EUSTERMAN, M.D. \\ ROCHFSTER, MINN.
}

This review is undertaken to emphasize proved clinical factors and briefly to consider various phases of experimental, clinical and therapeutic advancement in their application to the diagnosis of benign, chronic gastric and duodenal ulcers. Our observations are based on the study of 2,400 cases of gastric and duodenal ulcers operatively demonstrated in the Mayo Clinic from 1900 to 1914, inclusive, with an especial summary of the cases of 1913 and 1914.

\section{DIAGNOSIS}

A skilful anamnesis, in the absence of extensive clinical observations or direct roentgenologic evidence of a lesion, is still the most important factor in the differential diagnosis of lesions causing gastric disturbance. With regard to ulcer, characteristic chronicity or remission of symptoms is readily apparent in the case records in over 80 per cent. of all our proved cases. The principles emphasized by Graham $^{1}$ since his earliest observations are as effective today as then and are the accepted diagnostic criteria in a large daily experience. Pyloric and duodenal ulcers in which complications are not far advanced invariably manifest periodic exacerbations. Seasonal variations, especially of spring and fall, appear in almost 40 per cent. of all cases. Nervous and physical fatigue, infec-

* From the Mayo Clinic.

* Read before the Section on Practice of Medicine at the Sixty. Sixth Annual Session of the American Medical Association, San Fran. cisco, June, 1915

1. Graham, Christopher: Diagnosis Between Duodenal Ulcer and Gallstone Disease, ThE Journal A. M. A., Feb. 9, 1907, p. 515; Differential Diagnosis of Diseases Causing Gastric Disturbances, Northwestern Lancet, 1910, xxx, 139. tion and exposure, dietetic indiscretions, toxic intestinal disturbance, etc., are prominent factors influencing recurrences. Between seizures complete relief from symptoms is the rule although frequently in ulcers situated well up along the lesser curvature remission rather than periods of complete relief may occur. In general the greater number of these patients have had symptoms for from five to twenty years, in our experience, few less than one, and a constant average of twelve and one-half years. During the period of attack, pain or distress, variable in character and intensity, is the common and constant symptom in nearly all cases.

Of secondary diagnostic significance are the location, type and area of radiation of the pain. The time of pain and method of its control are of primary importance. The complex of chronicity and periodicity of attacks in which pain or distress and the usual association of symptoms repeated uniformly day by day during the attack and bearing a fairly definite relation to food intake and control is of primary clinical importance in the diagnosis of 80 per cent. of cases of uncomplicated peptic ulcers. In the remaining 20 per cent..this complex may be irregular, atypical or almost entirely absent; or the symptoms may be "mixed," owing to coincidence of disease in contiguous organs, which occurs in about 16 per cent. of all cases of ulcer. It is this group which often taxes the resources of the clinician, but errors in diagnosis do not easily occur if the possibilities are constantly borne in mind especially in cases (1) in which a perforative process or a painful spasm simulates hepatic colic, (2) in cases in which ulcers had become chronic and complicated when the first symptoms appeared, and (3) in cases suggesting a malignant process, the result of an extensive ulcer, hemorrhage, cachexia and perhaps a palpable inflammatory mass.

Clinical differentiation between gastric and duodenal ulcer is extremely difficult and in most instances wellnigh impossible. This has been the opinion of most observers. With great regularity late pain and the nature of hemorthage in proved gastric ulcer may suggest duodenal lesion. Then, too, not infrequently the earlier onset of pain in some cases of duodenal ulcer suggests gastric lesion. In about 5 per cent. of all ulcers coincident lesions in the stomach and duodenum are noted, an incidence which increases our difficulties. In our experience and in that of many other observers the Roentgen ray is of inestimable value. In this field it has its most practicable application besides occasionally furnishing the first reliable evidence to indicate the presence of ulcer; but too great reliance must not be placed on this ; for, valuable as may be the information afforded by the Roentgen examination, it has its limitations. The degree of demonstrability of gastric ulcer is directly concerned with three factors: that is, position, size and depth of the ulcer. The direct sign of irregularity of the gastric contour (niche, accessory pocket) may be wanting in instances in which the ulcer is situated on the anterior wall, high up in the cardia and when it involves the pyloric segment; should it be shallow, "slit-like," it may not be successfully demonstrated. ${ }^{2}$ Indirect and auxiliary signs, however, in combination with clinical data, may be helpful in establishing the diagnosis. The strikingly frequent occurrence of bulb deformity or hyperperi-

2. Carman, R. D.: The Diagnosis of Gastric Ulcer by the X-Ray, Read before the Kansas Medical Society, Kansas City, Kan., May 5, 1915. 
stalsis with residue in duodenal ulcer is worthy of note from both the point of diagnosis and localization.

The continuous or intermittent presence of altered or fresh blood in the gastric extract, the absence of evidence pointing to gross motor disturbances and the situation of pain and tender. point to the left of thie median line are strong presumptive evidence of gastric ulcer. Clinically, this general observation can be made: Uncomplicated types of duodenal and pyloric uker are invariably clear-cut throughout their life history; the longer the period between intake and pain, the lower the ulcer, as a rule; the more prompt the onset and the briefer the duration of pain, the higher the ulcer. In ulcer of the stomach proper this may not be so clear-cut as in duodenal or pyloric types, nor are the day-by-day symptoms so clearly defined. In ulcers well above the pylorus the symptoms are likely to be continuous, or remissions rather than intervals of complete relief are likely to be noted. The pain is not so often relieved by food; small amounts of food may give relief, while increased amounts may cause distress; more care as to diet is necessary; if bleeding occurs, hematemesis predominates; vomiting plays a more frequent rôle even in the absence of obstruction and affords relief; soda relieves pain when food does not; pain begins earlier, as a rule, often disappearing before the next meal, for obvious reasons, in the absence of stasis or marked hypersecretion. Thus the food-relief is minimized; but pain from one-half hour to two hours after food is quite the rule and is of diagnostic significance. Too much clinical significance cannot be placed on reliable evidence of gross hemorrhage in the presence of other symptoms characteristic of ulcer. This complication, however, occurs in only about 35 per cent. of all chronic gastric and duodenal ulcers. The association of hemorrhage with the ulcercomplex makes for safe diagnosis of ulcer in 95 per cent. of all cases. In 5 per cent. of all ulcers there is a silent bleeding type in which blood is regularly found in the stools but more or less complete absence of other usual clinical symptoms of pain, hyperacidity and food ease. Benign ulcer, unlike cancer, bleeds intermittently. Positive analysis of occult blood in the stool on limited examination was present in about 25 per cent. only of the cases studied. Hemorrhage has been noted in 2 per cent. of all cases of chronic appendicitis associated with marked gastric disturbance, while it is as high as 5 per cent. in chronic cholecystitis associated with the gastric reflex. In these cases the hemorrhage is probably due to primary follicular ulceration of the gastric or duodenal mucous membrane. More extensive observations on the frequency of occult blood, of general blood morphology and its diagnostic significance in ulcer are now under way and will be the subject of future consideration.

Briefly stated, in those organic conditions which most frequently cloud the diagnosis-chronic cholecystitis and cholelithiasis, chronic appendicitis, etc.we obtain our greatest diagnostic aid from the irregularity of symptoms during the period of attack. This irregularity is chiefly concerned with the time of appearance of pain and the influence of food. Nothing follows in sequence day by day, perhaps due to the fact that the stomach behaves properly unless irritated by the contiguous lesion and this extrinsic lesion is irregular in its influence. Judging from our case records, chronic catarrhal or "strawberry" cholecystitis very perfectly and most frequently simulates the ulcercomplex. The features of chronicity and "spells" (of invariably briefer duration, however) with characteristic pain, hyperacidity and flatulency having some food relation, the absence of colic, icterus and localizing signs are not uncommon. In order to lessen diagnostic error, daily clinical observation, repeated gastric analyses under variable circumstances, examination of the feces and, finally, the therapeutic management based on the well-known principles laid down by Sippy $^{3}$ may be necessary. It is unnecessary to comment on the importance of having in mind and recognizing these types of chronic painful dyspepsia most frequently having their origin in these extragastric lesions. Of considerable moment, too, is the frequency of lesions coexistent. in the gastroduodenal, biliary, pancreatic or appendiceal systems in which disturbances engendered by one may overshadow or make irregular the symptomatology of the other so that to the conceits of surgery must be left the ultimate diagnosis in too frequent instances.

Test-meal analysis so essential to gastric diagnosis has also its limitations. In the absence of definite evidence of blood, stasis, hypersecretion in the fasting contents, cardiac obstruction, etc., the findings are of value in so far as they are correlated with the clinical and roentgenologic data. They constitute a link, often a decisive one, in the chain of evidence. The instances of normal or subnormal acid value in the presence of a peptic ulcer occur in about 20 per cent. of all cases; on the other hand, hyperacidity and hypersecretion, even stasis of the first degree, are only too frequently associated with functional states, ptosis and extragastric lesions. These conditions would soon lead to diagnostic confusion if too much dependence were placed on a single or even a repeated gastric analysis. Again, on account of purely physical conditions a considerable residue may be overlooked as shown by the contribution of Harmer and Dodd. ${ }^{4}$ This disadvantage can be largely overcome by the use of a lavage tube of proper consistency, calibration and ample fenestration such as is now in common use. The researches of Rehfuss ${ }^{5}$ and his associates have made us revise our estimate of the normal contents of the fasting stomach. In selected cases the fractional study of gastric digestion by means of the same investigators' tube and methods has proved to be of practical diagnostic value. Hyperacidity is the rule in 60 per cent. of cases of gastric ulcer and hypersecretion, also in ulcers at or near the pylorus. These features, singly or combined, are noted in 75 per cent. of all duodenal ulcers. A considerable hypersecretion of 200 c.c. or more, likewise the recovery of retention contents of our modified Riegel meal after from twelve to fourteen hours invariably argues for a lesion or organic stenosis, especially if the findings are constant. The determination of gastric motility is of diagnostic importance second only to secretory function or disturbance and for obvious reasons the former is of especial interest to roentgenologists.

3. Sippy, Bertram W.: Diseases of the Stomach, Practical Treatment Musser and Kelly, W. B. Saunders Company, Philadelphia, 1912, ii 336; Gastric and Duodenal Ulcer: Medical Cure by an Efficient Remova of Gastric Juice Corrosion, The Journal A. M. A., May 15, 1915 p. 1625 .

p. 4. Harmer, T. W., and Dodd, W. J.: Sources of Error in the Use of the Stomach Tube for Diagnosis, Arch. Int. Med., November, 1913, p. 488

5. Rehfuss, M. E.; Bergeim, O., and Hawk, P. B.: Gastro-Intestinal Studies: I, The Question of the Residutum Found in the Empty Stomach, THE Journal A. M. A., July 4, 1914, p. 11; II, The Fractiona Study of Gastric Digestion with a Description of Normal and Patho. logic Curves, Tine Jourial A. M. A., Sept. 12, 1914, p. 909. 
Carman and Miller $^{8}$ conclude that their bariumized carbohydrate-meal method is a more sensitive test for gastric motility than the modified Riegel meal as commonly used in our clinic; that the modified Haudek double meal method is more informative than tubing after a motor-meal test since the former not only shows delay of evacuation beyond six hours but also yields information as to hypermotile conditions. Their conclusions are based on a comparison of results of both examinations in 950 patients who came to operation. The lesions were as follows: appendix, 125; gallbladder, 311 ; gastric ulcer, 109 ; gastric cancer, 137; duodenal ulcer, 268. Of these patients 220 (23.1 per cent.) showed a six-hour barium residue; 131 (13.7 per cent.) had food remnants after fourteen hours or longer. The majority (209, or 90.4 per cent.) of the 220 patients showing a barium retention were found to have cancer, or gastric or duodenal ulcer. They further conclude that a distinct residue after six hours from the barium meal given under prescribed conditions was in 90 per cent. of instances indicative of grave pathology, and usually denoted obstruction at or near the pylorus.

From personal observation we can concede a more sensitive test for motility in this method, especially in case of intermittent or low-grade retention dependent on pylorospasm, incomplete stenosis and on lesions of the accessory digestive system. One may add that the comparison of results between the two methods is hardly fair, principally because of difference in the time element and other features which must be taken into consideration. The motor meal under proper circumstances will continue to be a most practicable index of gastric motility - a fact conceded by eminent roentgenologists and emphasized by clinicians following observations made from the results of both methods. By the latter method one has the additional advantage of being able at the same time to estimate the secretory function of the stomach.

\section{STATISTICAL STUDY}

Exclusive of those cases in which a consistent diagnosis of gastric or duodenal ulcer was made and medically treated, there were 1,078 cases operatively demonstrated during the years 1913 and 1914. Of these, 264 were gastric and 814 were duodenal. Coexistent lesions in both organs were noted in 44 , or 4 per cent. The average ratio of gastric to duodenal ulcers is well shown in this series, about 1 to 3 , that is, 75 per cent. of the ulcers were duodenal. Of the 264 cases of gastric ulcer, 171 occurred in males and 93 in females. The average ages were 47 and 44.5 years, respectively. The average duration of symptoms was 9.8 years. In about 80 per cent. the course was intermittent, free intervals alternating with "spells" of variable duration, regular (40 per cent.) or irregular (49.5 per cent.) in frequency. In 50 per cent. the complaint was continuous and progressive over periods of from several months to several years prior to operation. Irrespective of the situation of the ulcer, pain appeared within 4 hours after meals in 85 per cent. of the cases; in over 30 per cent. it was present within 2 hours, in 55 per cent. within 3 hours, regarded as constant in 3.8 per cent. and irregular in 3.4 per cent. Definite nocturnal pain only occurred in 6 cases, or

6. Carman, R. D., and Miller, Albert: The Roentgenologic Determination of Gastric Motility with a Comparison of the Results Obtained in a Series of Cases Examined Both by the Roentgen Ray and the Test-Meal, Arch. Int. Med., to be published.
2 per cent. Pain was controlled by food or alkalies, or both, in 180 cases. The majority of these ( 84 per cent.) showed relief after food, while alkalies alone gave relief in 15.6 per cent. There was a fairly dérinite history of bleeding (hematemesis or melena) in 73 cases, or 27.6 per cent. of this group; about 41 per cent. had hematemesis only, 15 per cent. melena, while both hematemesis and melena were mentioned in 44 per cent.

Gastric analyses were carried out in 255 cases: 38 per cent. of these showed presence of altered blood in the extract. Gross retention was evident in 33.3 per cent. The average total acidity was 54 ; the average hydrochloric acid was 42 . In 18 per cent. the acid values were normal or below. The absence of free hydrochloric acid was shown in 13 cases. Under such a circumstance the possibility of carcinoma, syphilis or associated disease was evident. Five of these patients have since died from malignancy, three now apparently show malignancy; in the others the advanced age associated wth considerable fresh blood in the extract, gallbladder disease or other pathologic lesions explained the achlorhydria.

The generally accepted complex of ulcer was more or less definitely present in 81 per cent. of the 212 cases; irregular but suggestive in 6.8 per cent.; classified as irregular in 5.3 per cent. In the remaining 7 per cent. the picture was atypical or the record was incomplete. A primary diagnosis of gastric ulcer was made in 174 cases, or 66 per cent.; an alternative one in 21 cases ( 8 per cent.). Duodenal ulcer was the primary diagnosis in 47 cases ( 27 per cent.). The Roentgen ray gave definite assistance in 65 per cent. of the 113 cases examined in this series.

The situation of the ulcers was as follows: the lesser curvature in 167 cases, or 63 per cent.; the pylorus, 35 , or 13 per cent. ; total ulcers at or near the pylorus, 87 , or 33 per cent.; posterior wall, 27 , or 10 per cent.; anterior wall 6, or 2 per cent.; 2 gastrojejunal ulcers secondary to gastro-enterostomy. Multiple ulcers were noted in 15 cases, or 5.6 per cent.

The complications were: advanced cicatricial pyloric stenosis, 11.7 per cent.; evidence of perforation, 28 per cent. ; perigastritis with adhesions to neighboring structures, 28 per cent.; questionable malignancy, 11.7 per cent.; appendiceal disease, 33.7 per cent. In less than 25 per cent. of all cases was there no complicating factor, a fact which illustrates the advanced stage of the process at the time of first examination.

Of 814 cases of duodenal ulcers 628 (77 per cent.) were males and 186 (22.8 per cent.) were females. This characteristic disproportion in sex incidence Wilkie ${ }^{7}$ has probably explained on an anatomic basis. The average age was 43 years and the average duration of symptoms was over twelve years. The clinical course was intermittent in 95 per cent. of all cases, and periodic in 50 per cent. Continuous preoperative complaint of variable duration was noted in 26 per cent. In more than 85 per cent. the pain appeared in from two to five hours after taking food; in the remainder it appeared within two hours. Nocturnal pain only was given in 6 cases ( 7 per cent.). Definite relief by food was noted in 67 and partial or irregular relief in an additional 15 per cent. Relief by neutralization was present in 39 per cent. Hemorrhage was

7. Wilkie, D. P. B.: Observations on the Pathology and Etiology of Duodenal Ulcers, Edinburgh Med. Jour., 1914, xili, 196. 
reported in 208 cases ( 25 per cent.) and was classified: melena, 8.8 per cent. ; hematemesis, 6 per cent. both meiena and hematemesis, 10.5 per cent. The acid values averaged 20 per cent. higher than in gastric ulcer and in only 7 per cent. were they below the accepted normal standard.

A primary clinical diagnosis was made in 543 cases (66.7 per cent.) and an alternative one in 67 cases; that of primary gastric ulcer in 88 cases ( 10.8 per cent.). This totaled 85.7 per cent., the usual average in all the series recently studied. Of 251 cases in which the tentative diagnosis of gallbladder disease was also made, 51 (20 per cent.) showed this disease present and 51 ulcers (22.3 per cent.) were shown to be chronic perforating. Reviewing the case histories, the ulcer complex was fairly regular in 71 per cent., suggestive in 7.2 per cent., and irregular in 13.2 per cent.

At operation advanced pyloric stenosis was shown in 251 cases (30.8 per cent.); perforation in 26.8 per cent.; periduodenal inflammatory disease in 23.5 per cent. Associated disease in the appendix occurred in 48 per cent.; in the gallbladder in 9.7 per cent.

A more detailed comparative study of the statistical end-results in these two groups serves to bring out the salient differential clinical features as previously mentioned.

\section{ABSTRACT OF DISCUSSION}

Dr. J. N. Hall, Denver: We have the very great advantage of knowing what we are discussing, since the diagnosis has been proved in every case. There is perhaps no other common disease so frequently and so closely mimicked in its symptomatology by several other diseases as is peptic ulcer. We have all seen patients treated for twenty years for gastric ulcer, but curred by the removal of the chronically inflamed appendix, with no ulcer present. I have recently had one of Ewald's patients so cured, and it is doubtful if he is surpassed in diagnostic ability along this line by anyone in the world. Chronic inflammation of the gallbladder, chronic gastritis of the hyperacid type, small omental hernias and even the tabetic crises, are other common causes of confusion in diagnosis. Whenever ulcer is suggested but the history is atypical, the evidence as to the possible presence of chronic appendicitis should be reviewed, for such a condition will often be found. I should personally lay more stress on the search for unilateral muscular rigidity, which is probably of mofe decisive value as evidence of a dehnite structural lesion than pain and tenderness combined. In no procedure is the possession of an educated more imperative. The appearance in the anamnesis of jaundice and biliary colic has been the cause of diagnostic defeat in many cases through failure to recognize that in duodenal ulcer the patency of the common duct may be compromised, either mechanically or through an infective process. We should doubtless give the appendix more credit than it now receives as a prime cause of peptic ulcer, since the commonly induced hyperacidity in chronic appendicitis is probably a potent factor. It is interesting to note that the essayist found the appendix inflamed in 30 or 40 per cent. of the cases. If the ulcer does not heal promptly under proper medical measures, operation should certainly be advised. One comforting thought under these circumstances is that we shall save more than enough lives through the finding of unsuspected cancer to balance the mortality rate. The fact that many ulcers may be found by opening the stomach or duodenum when they would have escaped detection otherwise, should be given due consideration. There are so many chances of finding cancer, pyloric or duodenal obstruction, serious adhesions, hourglass deformity, etc., that we practically always find more atvanced pathologic changes than we anticipated. The acquirement of a drug habit or the development of arteriosclerosis, neurasthenic features or tuberculosis from the faulty nourishment must not be overlooked.

Dr. MAX EINHORN, New York: I have mentioned on another occasion the reason for the discrepancy between the views of the Mayos regarding the frequency of ulcer and the views of other clinicians. The Mayos believe ulcer is two or three times as frequent as cancer. I have no doubt their work supports that opinion. But there is a difference in the material that comes to the surgeon and the material which comes to the clinician. Say I have twenty cases of ulcer, probably only one of those will undergo operation. The cases which undergo operation belong to those in which there is food stagnation, or the lesion is near the pylorus, and that is why the surgeons find duodenal ulcer so frequently. If we could open up all the ulcer cases, we would probably find ulcer of the stomach much more frequent than ulcer of the duodenum. As to differential diagnosis, I agree with Dr. Eusterman, that from clinical symptoms we can sometimes make a diagnosis, but usually we cannot do so. In the great majority of cases we will be able to trake a more definite diagnosis on careful observation of the cases, especially as to the location of the ulcers. I have several cases in which the lesion was shown to bc in the duodenum, whereas the symptoms would indicate gallbladder trouble. There are of course cases in which the two conditions are present-duodenal ulcer and gallstones. This sometimes can be found out before operation by careful examination. The duodenal tube and the examination of the bile and duodenal contents may furnish a great deal of information as to the presence of gallbladder trouble. It has been my experience that in gallbladder disease the bile is not as clear as it should be when we take it out fresh from the duodenum.

Dr. J. A. Lichty, Pittsburgh: An essential factor und $: r$ this subject is the differentiation between the acute and chronic gastric and duodenal ulcers. The differences of opinion between the internists and the surgeons hinge largely on the failure to make this differentiation. The difference is just as great as between an acute ulcerative endocarditis and an old mitral lesion in a compensating heart. If we can differentiate definitely between the acute gastric and duodenal ulcer cases and the chronic cases with their consequent deformity or disturbance of function, we can very easily decide what are medical and what are surgical cases. A year ago before this section Dr. Joslin of Boston spoke of his results in gastric and duodenal ulcers. $\mathrm{He}$ found that most of his patients did not come to the internist until they had their disease six years, and they did not go to the surgeon until they had their disease ten years. It is useless for the physician and surgeon to accuse one another of neglect, etc., when in six years so much damage may have occurred that neither the physician nor the surgeon can expect satisfactory results.

The Reward of Scientific Study.-The student does not study Nature because that study is useful, but because it gives him pleasure, and it gives him pleasure because Nature is beautiful; if it were not beautiful it would not be worth knowing and life would not be worth living. I am not speaking, be it understood, of the beauty of its outward appearance-not that I despise it, far from it, but it has nothing to do with science: I mean that more intimate beauty which depends on the harmony in the order of the component parts of Nature. This is the beauty which a pure intelligence can appreciate and which gives substance and form to the scintillating impressions that charm our senses. Without this intellectual support the beauty of the fugitive dreams inspired by sensual impressions could only be imperfect, because it would be indecisive and always vanishing. It is this intellectual and self-sufficing beauty, perhaps more than the futtre welfare of humanity, that impels the scientific man to condemn himself to long and tedious studies.-H. Poincaré. 